\title{
On the Product Form of Quasi-Free States
}

\author{
J. Manuceau, F. Rocca ${ }^{\star}$ and D. Testard \\ Centre de Physique Théorique, Aix-Marseille University
}

Received October 21, 1968

\begin{abstract}
The product form of quasi-free states is outlined, and the types of the generated factors are exhibited whenever the states are translation invariant. Among these states some are shown to be involved in the study of Fermi and Bose gases.
\end{abstract}

\section{Introduction}

The "quasi-free states" originated from the "generalized free fields" introduced by $\mathrm{O}$. W. Greenbera [1]. They were defined and studied in references [2-7].

Whenever the quasi-free states of the $C^{*}$-algebra of commutation relations or of anticommutation relations are examined the papers [5] or $[6]$ are referred to.

In Section 2, the fermion case is considered; it is chiefly shown that any quasi-free state is, with the meaning given by Powers [3], a product state of partial states. These latter ones are primary if the state is translation-invariant and their types are exhibited.

An analogous analysis is made in Section 3 in the boson case, and similar results are obtained.

Finally we conclude by showing the physical significance of some quasi-free states involved in the study of Fermi and Bose gases.

\section{Fermions}

\subsection{Generalities}

Let $(H, s)$ be a real Hilbert space of finite or infinite, but countable dimension, equipped with a scalar product:

$$
(\psi, \varphi) \in H \times H \rightarrow s(\psi, \varphi) \in R
$$

(one-particle space). Consider the Clifford algebra $\mathfrak{A}(H, s)$ built on $(H, s)$; that is an involutive algebra with unit element (denoted by 1 ), and generated by the set of elements $B(\psi)$, linear with respect to $\psi$, which satisfy the anticommutation relations:

$$
[B(\psi), B(\varphi)]_{+}=2 s(\psi, \varphi) 1 .
$$

* Attaché de recherche au C.N.R.S. 
A unique norm can be found on $\mathfrak{A}(H, s)$, such that, after completion, $\overline{\mathfrak{A}(H, s)}$ becomes a $C^{*}$-algebra [7]. We shall note $\overline{\mathfrak{A}_{e}(H, s)}\left(\operatorname{resp} . \overline{\mathfrak{A}_{0}(H, s)}\right)$ the $C^{*}$-subalgebra (resp. the closed vector-subspace) of $\overline{\mathfrak{A}(H, s)}$ generated by products of even (resp. odd) number of $B(\psi)$ 's, and we shall call it "even part" (resp. "odd part") of $\overline{\mathfrak{A}(H, s)}$.

The following property holds:

$$
\overline{\mathfrak{A}(H, s)}=\overline{\mathfrak{A}_{e}(H, s)} \oplus \overline{\mathfrak{A}_{0}(H, s)} .
$$

A state $\omega$ on $\overline{\mathfrak{A}(H, s)}$ will be called quasi-free $[3-5]$, when:

together with:

$$
\omega \mid \overline{\mathfrak{A}_{0}(H, s)}=0
$$

$\omega\left(B\left(\psi_{1}\right) \ldots B\left(\psi_{2 n}\right)\right)=\sum(-1)^{p} \omega\left(B\left(\psi_{i_{1}}\right) B\left(\psi_{j_{1}}\right)\right) \ldots \omega\left(B\left(\psi_{i_{n}}\right) B\left(\psi_{j_{n}}\right)\right)$

the sum being extended to all two-by-two arrangement of $1,2, \ldots, 2 n$, such that $i_{k}<j_{k}, i_{1}<i_{2}<\cdots<i_{n} ;(-1)^{p}$ is the parity of the permutation $(1,2, \ldots, 2 n) \rightarrow\left(i_{1}, j_{1}, \ldots i_{n}, j_{n}\right)$.

Any quasi-free state determines an operator $A$ acting on $(H, s)$, which is antisymmetric of norm less than 1 and defined by:

$$
\omega(B(\psi) B(\varphi))=s(\psi, \varphi)+i s(A \psi, \varphi) .
$$

Conversely, any such operator $A$ determines by (2.1.3) a quasi-free state.

Let $\omega_{A}$ be such a state, and let $\pi_{A}, \mathfrak{S}_{A}$ and $\Omega_{A}^{-}$be respectively the representation, the representation space and the cyclic vector obtained from $\omega_{A}$ through the Gelfand-Segal theorem.

If $A^{2}=-1$, a complex structure is defined on $(H, s)$ through the relation:

$$
(\alpha+i \beta) \psi=\alpha \psi+\beta A \psi
$$

the right-hand side in (2.1.3) is then a scalar product which turns $H$ into a complex Hilbert space. Then $\omega_{A}$ is called "Fock state"; it is well known that such a state is pure.

If $A=0$, we are left with two cases:

$\operatorname{dim} H=2 n$ or $\infty$; then one can easily see by looking at (2.1.3) together with (2.1.2) that $\omega_{0}$ is the unique central state on $\overline{\mathfrak{P}(H, s)}$. It is a primary state of type $\mathrm{II}_{1}$ if $\operatorname{dim} H=\infty$ [8].

$\operatorname{dim} H=2 n+1 ; \omega_{0}$ is the unique central state to be quasi-free; it is not a primary state.

In the general case, the polar decomposition ${ }^{1}$ of $A$ is written in the following form:

$$
A=J|A|
$$

1 The theorem on the polar decomposition is shown to hold through the existence of the positive square root of a positive operator. Such a decomposition is still possible on a real Hilbert space. 
with $J$ such that $J^{2}=-1$ on $(\operatorname{ker} A)^{\perp}$. Through $J$, this space is provided with a complex structure. The operators $J$ and $|A|$ commute since $A$ is a normal operator.

Let $(H, s)$ be written as a Hilbert sum $H=\sum_{n \in I}^{\oplus} H_{n}(I \subset N)$, and let $\omega_{n}$ be $\omega$ when restricted to $\overline{\mathfrak{A}\left(H_{n}, s\right)}$ ( $\omega$ a quasi-free state). $\omega$ will be called a product state [3] with respect to such a sum if for any $(n, m) \in I \times I$, the following relation holds:

$$
\omega(X Y)=\omega(X) \omega(Y) \text { for any } X \in \overline{\mathfrak{A}\left(H_{n}, s\right)}, \quad Y \in \overline{\mathfrak{A}\left(H_{m}, s\right)} .
$$

Accordingly, we shall write:

$$
\omega=\dot{\bigotimes}_{n \in I} \omega_{n}
$$

\subsection{On the Product Form of Quasi-Free States}

(2.2.1) Lemma. Let $\left(H_{n}\right)_{n \in I}$ a sequence of orthogonal subspaces of $(H, s)$ invariant under $A$ and such that $H$ be their Hilbert sum. $\omega_{A}$ is a product state:

where $A_{n}$ is $A$ restricted to $H_{n}$.

$$
\omega_{A}=\dot{\otimes}_{n \in I} \omega_{A_{n}}
$$

From linearity and continuity, it is sufficient to prove that:

when

$$
\omega_{A}(X Y)=\omega_{A}(X) \omega_{A}(Y)
$$

$$
X=B\left(\psi_{1}\right) B\left(\psi_{2}\right) \ldots B\left(\psi_{q}\right), \quad Y=B\left(\varphi_{1}\right) B\left(\varphi_{2}\right) \ldots B\left(\varphi_{m}\right)
$$

with $\psi_{p} \in H_{n}, p=1, \ldots, q$ and $\varphi_{k} \in H_{n}^{\perp}, k=1, \ldots, m$. When the parities of $q$ and $m$ are not the same (2.1.1) must be used. When they are the same, by rewriting (2.1.2) into:

$$
\omega_{A}(X Y)-\omega_{A}(X) \omega_{A}(Y)
$$

a sum of product terms is obtained in which one term at least is such as the following:

$$
\omega_{A}(B(\psi) B(\varphi))
$$

with $\psi \in H_{n}$, and $\varphi \in H_{n}^{\perp}$, and consequently is vanishing from (2.1.3) and the hypothesis.

(2.2.2) Theorem. Any quasi-free state $\omega_{A}$ is a product state with respect to the decomposition of $(H, s)$ into a Hilbert sum $H=H_{1} \oplus H_{2} \oplus H_{3}$, with

$$
\begin{aligned}
& H_{1}=\operatorname{ker}(1-|A|) \\
& H_{2}=\operatorname{ker} A \\
& H_{3}=H \ominus\left(H_{1} \oplus H_{2}\right) \text { with } A=J|A| .
\end{aligned}
$$


$H_{1}, H_{2}, H_{3}$ are clearly invariant under $A$ and they are orthogonal to each other; we obtain the result from the preceding lemma. Consequently:

$$
\omega_{A}=\omega_{A_{1}} \dot{\otimes} \omega_{A_{2}} \dot{\otimes} \omega_{A_{3}} .
$$

From the hypothesis, $A_{1}^{2}=-1$ and $A_{2}=0$, the state $\omega_{A_{1}}$ is a Fock state and $\omega_{A_{2}}$ is the central state.

(2.2.3) Theorem. The pure quasi-free states are precisely the Fock states.

It is necessary and also sufficient for $\omega_{A}$ be pure that $\omega_{A_{n}}$ also be pure [3]. Consequently one needs only the following lemma.

(2.2.4) Lemma. If $H_{1}=H_{2}=\{0\}, \omega_{A}$ is not pure.

Let us set:

$$
T_{1}=\frac{1}{\sqrt{2}}(1+|A|)^{1 / 2} \quad T_{2}=\frac{1}{\sqrt{2}}(1-|A|)^{1 / 2} .
$$

If $\Theta_{J}$ is defined on $\mathfrak{G}_{J}$ by:

with

$$
\left[\Theta_{J}, \pi_{J}(B(\psi))\right]_{+}=0 \quad \text { for any } \quad \psi \in H
$$

$$
\Theta_{J} \Omega_{J}=\Omega_{J}
$$

an easy calculation shows the equivalence between $\pi_{A}$ and the representation $\pi$ on $\mathfrak{G}_{J} \otimes \mathfrak{G}_{-J}$ defined by:

$$
\pi(B(\psi))=\pi_{J}\left(B\left(T_{1} \psi\right)\right) \otimes 1+\Theta_{J} \otimes \pi_{-J}\left(B\left(T_{2} \psi\right)\right)
$$

$\Omega_{J} \otimes \Omega_{-J}$ is a cyclic vector for this representation, since $T_{1}$ and $T_{2}$ are one to one, and the corresponding state is precisely $\omega_{A}$ on the product terms $B(\psi) B(\varphi)$. As it is easily shown [9] this state is quasi-free and consequently is precisely $\omega_{A}$. The representation $\pi^{\prime}$ defined by:

$$
\pi^{\prime}(B(\psi))=\Theta_{J} \pi_{J}\left(B\left(T_{2} \psi\right)\right) \otimes \Theta_{-J}-1 \otimes \Theta_{-J} \pi_{-J}\left(B\left(T_{1} \psi\right)\right)
$$

has precisely $\Omega_{J} \otimes \Omega_{-J}$ as its cyclic vector, and commutes with $\pi$. Consequently $\pi$ is not irreducible and $\omega_{A}$ is not a pure state.

\subsection{On the Type of Invariant Quasi-Free States}

(2.3.1) Lemma. For any vector subspace $E$ in $H$, we denote by $\mathfrak{A}(E, s)^{c}$ the commutant of $\mathfrak{A}(E, s)$ in $\overline{\mathfrak{A}(H, s)}$. If $\operatorname{dim} E=2 \mathrm{~m}$ :

$$
\mathfrak{A}(E, s)^{c}=\overline{\mathfrak{A}_{e}\left(E^{\perp}, s\right)} \oplus \Theta_{E} \overline{\mathfrak{A}_{0}\left(E^{\perp}, s\right)}
$$

with $\Theta_{E}=B\left(\psi_{1}\right) \ldots B\left(\psi_{2 m}\right)$ for any basis $\psi_{1}, \ldots, \psi_{2 m}$ of $E$.

It is straightforward that $\overline{\mathfrak{A}_{e}\left(E^{\perp}, s\right)} \oplus \Theta_{E} \overline{\mathfrak{A}_{0}\left(E^{\perp}, s\right)} \subset \mathfrak{A}(E, s)^{c}$. In order to obtain the inclusion relation in the other way, it must be noticed that:

$$
\mathfrak{A}(E, s)^{c} \cap \mathfrak{A}(H, s) \subset \mathfrak{A}_{e}\left(H^{\perp}, s\right) \oplus \Theta_{E} \mathfrak{A}_{0}\left(E^{\perp}, s\right) .
$$

Let us consider the elements $X=B\left(\varphi_{1}\right) \ldots B\left(\varphi_{p}\right)$ where $\varphi_{i}$ is either a vector belonging to the basis $\left\{\psi_{j}\right\}$ of $E$, or a vector belonging to $E \perp$. It must be shown that if $X$ belongs to $\mathfrak{A}(E, s)^{c}$, then it necessarily belongs to $\mathfrak{A}_{e}\left(E^{\perp}, s\right)$, or to $\Theta_{E} \mathfrak{A}_{0}\left(E^{\perp}, s\right)$ : 
if $p=2 n$, and if some $i$ can be found with $1 \leqq i \leqq 2 n$, such that $\varphi_{i} \in E, B\left(\varphi_{i}\right)$ anticommutes with $X$, that is contrary to the hypothesis. Consequently $X \in \mathfrak{A}_{e}\left(E^{\perp}, s\right)$

if $p=2 n+1$, and if some $i$ can be found, with $1 \leqq i \leqq 2 m$, such that $\psi_{i}$ coincides with no $\varphi_{j}, j=1, \ldots, 2 n+1$, then $B\left(\psi_{i}\right)$ anticommutes with $X$. Consequently $X \in \Theta_{E} \mathfrak{A}_{0}\left(E^{\perp}, s\right)$, and the result is proved.

The mapping $B(\psi) \rightarrow-B(\psi)$ can be extended to a unique automorphism $\gamma$ of the $C^{*}$-algebra $\overline{\mathfrak{A}(H, s)}$. This automorphism is such that:

$$
\begin{aligned}
& \gamma(X)=X \quad \text { if } \quad X \in \overline{\mathfrak{R}_{e}(H, s)}, \\
& \gamma(X)=-X \quad \text { if } \quad X \in \overline{\mathfrak{R}_{0}(H, s)} .
\end{aligned}
$$

Let $\gamma_{e}=\frac{1}{2}(1+\gamma)$ and $\gamma_{0}=\frac{1}{2}(1-\gamma)$ respectively the projection operators on the even and odd parts of $\overline{\mathfrak{A}(H, s)}$.

From (2.1.1) the quasi-free states $\omega_{A}$ are invariant under $\gamma$. Consequently a unitary operator $U$ can be found on $\mathfrak{S}_{A}$, defined by ([11], 2.12.11):

$$
\begin{aligned}
U \Omega_{A} & =\Omega_{A} \\
\pi_{A}(\gamma(X)) & \left.=U \pi_{A}(X) U \text { with } \quad U^{*}=U^{-1}=U .\right\}
\end{aligned}
$$

The operator $U$ can be used to define complementary projection operators $Q_{e}$ and $Q_{0}$ on the even and odd parts of $\mathfrak{L}\left(\mathfrak{S}_{A}\right)$ by:

$$
\begin{aligned}
& Q_{e}(V)=\frac{1}{2}(1+U V U)=V_{e}, \\
& Q_{0}(V)=\frac{1}{2}(1-U V U)=V_{0} \text { for any } V \in \mathbb{L}\left(\mathfrak{S}_{A}\right) .
\end{aligned}
$$

We get:

$$
\begin{aligned}
& \pi_{A}\left(\gamma_{e}(X)\right)=Q_{e}\left(\pi_{A}(X)\right), \\
& \pi_{A}\left(\gamma_{0}(X)\right)=Q_{0}\left(\pi_{A}(X)\right) .
\end{aligned}
$$

(2.3.3) Lemma. Using the notations of Lemma (2.3.1), we get for any quasi-free state $\omega_{A}$.

$$
\left.\left\{\pi_{A}\left(\mathfrak{U}(E, s)^{c}\right)\right\}^{\prime \prime}=\left\{\pi_{A}\left(\overline{\mathfrak{A}_{e}\left(E^{\perp}, s\right)}\right)\right\}^{\prime \prime} \oplus \overline{\pi_{A}\left(\Theta_{E} \overline{\mathfrak{A}_{0}\left(E^{\perp}, s\right)}\right.}\right) w
$$

with $\overline{\pi_{A}(\mathfrak{R})^{w}}$ denoting the weak closure of $\pi_{A}(\mathfrak{R})$.

From Lemma (2.3.1) it is straightforward to obtain including relation:

$$
\left.\left\{\pi_{A}\left(\mathfrak{U}(E, s)^{c}\right)\right\}^{\prime \prime} \supset\left\{\pi_{A}\left(\overline{\mathfrak{A}_{e}\left(E^{\perp}, s\right)}\right)\right\}^{\prime \prime} \oplus \overline{\pi_{A}\left(\Theta_{E} \overline{\mathfrak{U}_{0}\left(E^{\perp}, s\right)}\right.}\right)^{w} .
$$

On the other hand a sequence $\left(\pi_{A}\left(X_{n}\right)\right)_{n \in N}$ in $\pi_{A}\left(\mathfrak{Z}(E, s)^{c}\right)$ can be found converging to any $V \in\left\{\pi_{A}\left(\mathfrak{A}(E, s)^{c}\right)\right\}^{\prime \prime}$, in the weak topology. We can deduce from the above that $V+U V U=2 Q_{e}(V)$ is in the weak closure of $2 Q_{e}\left(\pi_{A}\left(X_{n}\right)\right)=\pi_{A}\left(X_{n}\right)+U \pi_{A}\left(X_{n}\right) U$, and analogously that $Q_{0}(V)$ is in the weak closure of $Q_{0}\left(\pi_{A}\left(X_{n}\right)\right)$. So $V \in\left\{\pi_{A}\left(\overline{\mathfrak{A}_{e}\left(E^{\perp}, s\right)}\right)\right\}^{\prime \prime}$ $\oplus \overline{\pi_{A}\left(\Theta_{E} \overline{\mathfrak{A}_{0}\left(E^{\perp}, s\right)}\right)}$. 
(2.3.4) Lemma. For any $X \in \overline{\mathfrak{2}(H, s)}$, an even-dimensional vector subspace $E$ of $H$ can be found, such that, for any $Y \in \overline{\mathfrak{2}_{e}\left(E^{\perp}, s\right)}$, the following relation holds:

$$
\left|\omega_{A}(X Y)-\omega_{A}(X) \omega_{A}(Y)\right| \leqq\|Y\| .
$$

For any $X \in \overline{\mathfrak{A}(H, s)}$, some $X_{0} \in \mathfrak{A}(H, s)$ can be found, with $\left\|X-X_{0}\right\| \leqq \frac{1}{2}$. Consequently:

and

$$
\left|\omega_{A}(X Y)-\omega_{A}\left(X_{0} Y\right)\right| \leqq \frac{1}{2}\|Y\|
$$

$$
\left|\omega_{A}(X) \omega_{A}(Y)-\omega_{A}\left(X_{0}\right) \omega_{A}(Y)\right| \leqq \frac{1}{2}\|Y\|, \text { for any } Y \in \overline{\mathfrak{A}(H, s)} .
$$

The result will be shown by exhibiting, for any $X_{0}$ such as $B\left(\psi_{1}\right) \ldots B\left(\psi_{n}\right)$, an even-dimensional subspace $E$ of $H$ such that, for any $Y \in \mathfrak{A}_{0}\left(E^{\perp}, s\right)$,

$$
\omega_{A}\left(X_{0} Y\right)-\omega_{A}\left(X_{0}\right) \omega_{A}(Y)=0 .
$$

We can take any even-dimensional subspace including $\psi_{1}, \psi_{2}, \ldots, \psi_{n}$, together with $A \psi_{1}, A \psi_{2}, \ldots, A \psi_{n}$. Using continuity and linearity we obtain the result by proving the relation for any product $Y=B\left(\varphi_{1}\right) \ldots . B\left(\varphi_{2 q}\right)$ in $\mathfrak{A}_{0}\left(E^{\perp}, s\right)$, which can be derived from the definition of $E$, through (2.1.2) and (2.1.3).

(2.3.5) Proposition. For any quasi-free state $\omega_{A}$ the center $\delta_{A}$ of $\left\{\pi_{A}(\mathfrak{U}(H, s))\right\}^{\prime \prime}$ is at most two-dimensional. More precisely, if $\operatorname{dim} \beta_{A}=2$, this center is generated by an odd hermitian operator $z$ satisfying $z^{2}=1 .^{2}$

Let $\mathscr{E}$ be the set of even-dimensional subspaces of $H$, we get from ([12], Lemma 2.4) and Lemma (2.3.3):

$$
\begin{aligned}
B_{A} & =\bigcap_{E \in \mathscr{E}}\left\{\pi_{A}\left(\mathfrak{Q}(E, s)^{c}\right)\right\}^{\prime \prime} \\
& =\bigcap_{E \in \mathscr{E}}\left\{\pi_{A}\left(\overline{\mathfrak{A}_{e}\left(E^{\perp}, s\right)}\right)\right\}^{\prime \prime} \otimes \bigcap_{E \in \mathscr{E}} \overline{\pi_{A}\left(\Theta_{E}{\overline{\mathfrak{A}_{0}\left(E^{\perp}, s\right)}}^{w}\right.} .
\end{aligned}
$$

The even (resp. odd) elements in the center belong to $\bigcap_{E \in \mathscr{E}}\left\{\pi_{A}\left(\mathfrak{A}_{e}\left(E^{\perp}, s\right)\right)\right\}^{\prime \prime}$ [resp. $\left.\overline{\pi_{A}\left(\Theta_{E} \overline{\mathfrak{A}_{0}\left(E^{\perp}, s\right)}\right)^{w}}\right]$. Using Lemma (2.3.4), one shows just like in ([12], Theorem 2.5 (iii) $\rightarrow$ (i)) that the even elements in the center are multiples of the identity. If $\operatorname{dim} \beta_{A}>1$, i.e. $Q_{0}\left(\delta_{A}\right) \neq\{0\}$, an odd hermitian element $z$ can be found in $\beta_{A} \cdot z^{2}$ being even and in the center, $z^{2}$ is $\mu 1$ with $\mu$ some positive non-vanishing number. Taking $\mu^{-1 / 2} z$ instead of $z$, we are brought back to the case $z^{2}=1$. For any $z^{\prime} \in Q_{0}\left(\delta_{A}\right)$, $z z^{\prime}$ is even and consequently written as some $\lambda 1$. It follows $z^{\prime}=\lambda z$.

Particular case of quasi-free translation invariant states allows us to make these results more precise.

${ }^{2}$ It has been recently shown by F. Rocca, M. Sirugue, D. Testard, and M. Wrnnink that $\omega_{A}$ is primary $\left(\operatorname{dim} \delta_{A}=1\right)$ if and only if $\operatorname{dim}(\operatorname{Ker} A)$ is odd. 
From now on $H=\mathfrak{Q}^{2}\left(R^{3}\right), s$ is the real part of the usual scalar product. The translation group $\left\{T_{x} \mid x \in R^{3}\right\}$ is an orthogonal group when equipped with $s$, inducing a group of automorphisms $\left\{\tau_{x} \mid x \in R^{3}\right\}$ of $\overline{\mathfrak{A}(H, s)}$, defined by [7]:

$$
\tau_{x} B(\psi)=B\left(T_{x} \psi\right) \text { for any } \psi \in \mathfrak{L}^{2}\left(R^{3}\right) .
$$

From the strong continuity of the mapping $x \rightarrow T_{x}$, the continuity of the mapping $x \rightarrow \tau_{x}(X)$ follows, for any $X \in \overline{\mathfrak{A}(H, s)}$.

(2.3.6) Proposition. Let $\omega_{A}$ be a translation-invariant quasi-free state. Using the notations of Theorem (2.2.2), $\mathrm{H}_{1}, \mathrm{H}_{2}, \mathrm{H}_{3}$ either have a zero dimension, or an infinite one.

It is known that $\left[A, T_{x}\right]_{-}=0$ for any $x([5]$, Lemma 2$)$ and so, since the polar decomposition of $A$ is unique, $T_{x}$ commutes with $|A|$. Consequently the spaces $H_{1}, H_{2}, H_{3}$ are invariant for $T_{x}$ and the proposition is deduced from the following lemma:

(2.3.7) Lemma. Any translation invariant subspace of $\mathfrak{Q}^{2}\left(R^{3}\right)$ has either a zero dimension, or an infinite one.

Let $\psi \in \mathfrak{L}^{2}\left(R^{3}\right)$ be such that the subspace $\left\{T_{x} \psi \mid x \in R^{3}\right\}$ is finitedimensional. Then the function $x \rightarrow\left(\psi, T_{x} \psi\right)$ is an almost-periodic function $([11], 16.2 .2)$, which, on the other hand, belongs to $\mathfrak{F}^{0}\left(R^{3}\right)$ (the set of continuous functions vanishing at infinity ([13], 14.10.7)). It follows $\psi=0([14], \S 24)$.

(2.3.8) Theorem. With the same notations as in (2.2.2), any translationinvariant quasi-free state is written as

with:

$$
\omega_{A}=\omega_{A_{1}} \dot{\otimes} \omega_{A_{2}} \dot{\otimes} \omega_{A_{3}}
$$

(i) $\omega_{A_{1}}$ is pure of type $I_{\infty}$.

(ii) $\omega_{A_{2}}$ is primary of type $I I_{1}$.

(iii) $\omega_{A_{3}}$ is primary of type $I I I$.

Since, from Proposition (2.3.6), the dimensions of $H_{1}$ and $H_{2}$ are vanishing or infinite, $\omega_{A_{1}}, \omega_{A_{2}}$ have respectively the type $I_{\infty}, I_{1}$, whenever they exist. Since $\omega_{A_{3}}$ is translation-invariant too, only the case with $H_{1}=H_{2}=\{0\}$ must be looked at. The following lemma will be needed:

(2.3.9) Lemma. Any quasi-free state is such that: $\omega_{A}\left(X \tau_{x}(Y)\right)-\omega_{A}(X) \omega_{A}(Y) \rightarrow 0$ with $|x| \rightarrow \infty, X, Y \in \overline{\mathfrak{A}(H, s)}$.

The property must be shown when $X$ and $Y$ are finite products of $B(\psi)$ 's, and it will be stated in any case from (2.1.2), (2.1.3), by noting that, for any $\psi$ and $\varphi \in \mathbb{Q}^{2}\left(R^{3}\right), s\left(\psi, T_{x} \varphi\right) \rightarrow 0$ when $|x| \rightarrow \infty$.

Since $\omega_{A}$ is translation invariant, we know that there exists a strongly continuous representation $U$, of $R^{3}$ into $\mathfrak{S}_{A}$, such that

$$
\pi_{A}\left(\tau_{x} X\right)=U(x) \pi_{A}(X) U(x)^{*} \text { with } U(x) \Omega_{A}=\Omega_{A} .
$$

The preceding lemma and ([15], Theorem 2) show that $\Omega_{A}$ is the unique invariant vector by the whole set of $U(x)$ 's.

4 Commun.math. Phys., Vol. 12 
It has been noticed in Lemma (2.2.4) that the representation defined through (2.2.6) had $\Omega_{A}$ as cyclic vector. $\Omega_{A}$ is, a fortiori, cyclic for the commutant of $\pi_{A}(\overline{\mathfrak{Q}(H, s)})$ and consequently separating for $\left\{\pi_{A}(\overline{\mathfrak{A}(H, s)})\right\}^{\prime \prime}$. Since the hypotheses of Theorem 2.4 in [16] are verified, either $\omega_{A}$ is a primary state of type $I I I$, or the center $\delta_{A}$ has no minimal projection operators. By Proposition (2.3.6), if $\omega_{A}$ is not primary, $\delta_{A}$ is generated by an hermitian operator $z$, satisfying $z^{2}=1$. There are $\frac{1}{2}(1+z)$ and $\frac{1}{2}(1-z)$ as projection operators in $\beta_{A}$, and nothing more. So $\omega_{A}$ is primary of type III.

(2.3.10) Remark. The Lemma (2.2.4) remains true when $\operatorname{dim} H_{2}=\infty$. Indeed it is possible to extend the complexification $J$ of $H_{3}$ to a complexification of $H_{2} \oplus H_{3}$. In the proof of (iii), the hypothesis $H_{2}=\{0\}$ was not needed. So $\omega_{A_{z}} \dot{\otimes} \omega_{A_{3}}$ is primary of type III.

(2.3.11) Theorem. Any translation-invariant quasi-free state $\omega_{A}$ is primary. With the same notations as in (2.2.2), we get:

(i) if $H_{3}=H_{2}=\{0\}, \omega_{A}$ is pure of type $I_{\infty}$,

(ii) if $H_{1}=H_{3}=\{0\}, \omega_{A}$ is a state of type $I I_{1}$,

(iii) if $H_{3}=\{0\}, H_{1}$ and $H_{2} \neq\{0\}, \omega_{A}$ is a state of type $I I_{\infty}$,

(iv) if $H_{3} \neq\{0\}, \omega_{A}$ is a state of type III.

(i) and (ii) are already known (2.3.8). Proofs of (iii) and (iv) will follow, using ([10], p. 102, Corollary 3 ) and (2.3.10), from the following lemma:

(2.3.12) Lemma. If $\omega_{A}=\omega_{A_{1}} \dot{\otimes} \omega_{A_{2}}$ is a product state with respect to the sum $H=H_{1} \oplus H_{2}$ and $\omega_{A_{1}}$ is pure and invariant under $\gamma$ (2.3.2), the Von Neumann algebra generated by $\pi_{A}(\overline{\mathfrak{A}(H, s)})$ is spatially isomorphic with the tensor product of the Von Neumann algebras generated by $\left.\pi_{A_{1}} \overline{\left(\mathfrak{A}\left(H_{1}, s\right)\right.}\right)$ and $\pi_{A_{2}}\left(\overline{\mathfrak{Q}\left(H_{2}, s\right)}\right)$. Hence $\omega_{A}$ is primary if $\omega_{A_{2}}$ is primary ([17], Prop. 1.6).

The representation $\pi_{A}$ is unitarily equivalent to the representation $\pi$ in $\mathfrak{H}_{A_{1}} \otimes \mathfrak{S}_{A_{2}}$ defined by:

$$
\begin{array}{lll}
\pi(B(\psi))=\pi_{A_{1}}(B(\psi)) \otimes 1 & \text { if } & \psi \in H_{1}, \\
\pi(B(\psi))=U \otimes \pi_{A_{2}}(B(\psi)) & \text { if } & \psi \in H_{2}
\end{array}
$$

where $U$ [see (2.3.2)] is an operator anticommuting with each $\pi_{A_{1}}(B(\psi))$, $\psi \in H_{1}$. Indeed the vector $\Omega_{A_{1}} \otimes \Omega_{A_{2}}$ is cyclic for $\pi$, and the corresponding state is precisely $\omega_{A}$. The lemma is at once deduced since:

$$
U \in\left\{\pi_{A_{1}}\left(\mathfrak{A}\left(H_{1}, s\right)\right)\right\}^{\prime \prime}=\mathfrak{L}\left(\mathfrak{S}_{A_{1}}\right) \text { and } U^{2}=1 .
$$

(2.3.13) Theorem ${ }^{3}$. Two translation invariant quasi-free states $\omega_{A}$ and $\omega_{A}$, are quasi-equivalent, if and only if $A=A^{\prime}$.

3 E. Størmer private communication. 
From the theory of asymptotically abelian $C^{*}$-algebras [15], it is known that $\omega_{A}$ is an extremal translation invariant state. Therefore, by ([18], Theorem 6.1), $\omega_{A}$ and $\omega_{A^{\prime}}$, are quasi-equivalent iff $\omega_{A}=\omega_{A^{\prime}}$, hence iff $A=A^{\prime}$.

\section{Bosons}

\subsection{The $C^{*}$-Algebra of Commutation Relations}

The $C^{*}$-algebra of commutation relations $\overline{\Delta(H, \sigma)}$ is studied in [19]. Let us simply recall some useful definitions and results.

$(H, \sigma)$ is a symplectic space, i.e. a real vector space (one-particle space) equipped with an antisymmetric, regular and bilinear form $\sigma$ (regularity means: $\sigma(\psi, \varphi)=0$ for any $\psi \in H$ implies $\varphi=0) . \Delta(H, \sigma)$ is the involutive algebra generated by unitary elements denoted by $\delta_{\psi}$, $\psi \in H$, verifying:

$$
\begin{aligned}
\left(\delta_{\psi}\right)^{*} & =\delta_{-\psi} \\
\delta_{\psi} \delta_{\varphi} & =e^{-i \sigma(\psi, \varphi)} \delta_{\psi+\varphi},
\end{aligned}
$$

$\delta_{0}$ is the unit of this algebra.

The set $\mathfrak{R}(H, \sigma)$ of representations of commutation relations is defined as the set of representations $\pi$ of $\Delta(H, \sigma)$ such that the mapping $\lambda \in R \rightarrow \pi\left(\delta_{\lambda_{\psi}}\right)$ be weakly continuous for any $\psi \in H$. By all these representations, a unique norm can be induced on $\Delta(H, \sigma)$ [i.e. for any $\pi_{1}$ and $\pi_{2} \in \mathfrak{R}(H, \sigma),\left\|\pi_{1}(X)\right\|=\left\|\pi_{2}(X)\right\|$, for any $\left.X \in \Delta(H, \sigma)\right]$. The closure of $\Delta(H, \sigma)$ with respect to this norm is the $C^{*}$-algebra $\overline{\Delta(H, \sigma)}$. For any $\pi \in \mathfrak{R}(H, \sigma): \pi\left(\delta_{\psi}\right)=e^{i B(\psi)}$, with $B(\psi)$ the field operator.

Let $H_{1}$ and $H_{2}$ be two subspaces which are regular in $(H, \sigma)$ (i.e. $\sigma \mid H_{1} \times H_{1}$ and $\sigma \mid H_{2} \times H_{2}$ are still regular $)$. Suppose $\sigma\left(\psi_{1}, \psi_{2}\right)=0$, for any $\psi_{1} \in H_{1}, \psi_{2} \in H_{2}$; then, if $H=H_{1} \oplus H_{2}$ :

$$
\overline{\Delta(H, \sigma)}=\overline{\Delta\left(H_{1}, \sigma\right)} \otimes \overline{\Delta\left(H_{2}, \sigma\right)} .
$$

A bijective operator $T$ on $(H, \sigma)$ will be said to be symplectic, if the following law holds:

$$
\sigma(T \psi, T \varphi)=\sigma(\psi, \varphi), \quad \psi, \varphi \in H .
$$

Then the mapping:

$$
\delta_{\psi} \rightarrow \delta_{T \psi}, \quad \psi \in H
$$

can be extended into a unique automorphism $\tau_{T}$ of $\overline{\Delta(H, \sigma)}$.

Analogously, for any element $\chi$ in the algebraic dual of $H$, the mapping:

$$
\delta_{\psi} \rightarrow e^{i \chi(\psi)} \delta_{\psi}, \quad \psi \in H
$$

can be extended into a unique automorphism $\xi_{x}$ of $\overline{\Delta(H, \sigma)}$, this latter being called "gauge automorphism of second kind induced by $\chi$ ". 


\subsection{On Real Scalar Products Defined on $(H, \sigma)$}

$H$ is equipped with the uniform structure defined by the following set of semi-norms:

$$
\varrho_{\varphi}: \psi \rightarrow|\sigma(\psi, \varphi)| .
$$

From now on we suppose $H$ to be sequentially closed, i.e. we suppose that any sequence $\left(\varphi_{n}\right)_{n \in N}$ of elements in $H$, such that, for any $\psi \in H$, $\left(\sigma\left(\psi, \varphi_{n}\right)\right)_{n \in N}$ is a Cauchy-sequence, converges to an element of $H$. On the other hand, let $\subseteq$ be the set of real scalar products $s$ on $H$ such that:

(3.2.1 a) $|\sigma(\psi, \varphi)|^{2} \leqq\|\psi\|_{s}^{2}\|\varphi\|_{\delta}^{2}$, with $\|\psi\|_{s}^{2}=s(\psi, \psi)$.

$(3.2 .1 \mathrm{~b}) \sigma$, when extended through continuity, into $\sigma^{\prime}$ on $\bar{H}^{s}$ (the closure of $H$ with respect to the norm $\left.\|\cdot\|_{s}\right)$ is regular. $(H, s)$ is then a real prehilbert space equipped with the norm $\|\cdot\|_{s}$. We shall denote by $\|\cdot\|_{s}$ the norm of bounded operators on $(H, s)$.

(3.2.2) Theorem. For any $s \in \mathcal{E},(H, s)$ is a real Hilbert space.

Let $\psi_{1}$ be in $\bar{H}^{s}$, a sequence $\left(\varphi_{n}\right)_{n \in N}$ can be found in $H$, converging to $\psi_{1}$ with respect to $\|\cdot\|_{s}$. Consequently (3.2.1) for any $\xi \in H$,

$$
\lim _{n \rightarrow \infty} \sigma^{\prime}\left(\varphi_{n}, \xi\right)=\sigma^{\prime}\left(\psi_{1}, \xi\right) \text {. }
$$

Since $H$ is sequentially closed, (3.2.1 a) implies that a $\psi_{2}$ can be found in $H$ such that, for any $\zeta \in H$,

$$
\lim _{n \rightarrow \infty} \sigma\left(\varphi_{n}, \zeta\right)=\sigma\left(\psi_{2}, \zeta\right)
$$

It follows that $\sigma^{\prime}\left(\psi_{1}-\psi_{2}, \zeta\right)=0$ for any $\zeta$ in $H$. From the continuity (3.2.1 a) and the regular character (3.2.1b) of $\sigma^{\prime}$, it follows that $\psi_{1}=\psi_{2} \in H$.

For any $s \in \mathfrak{S}$, there is a bounded operator $D_{s}$ on $H$ with $\left\|D_{s}\right\|_{s} \leqq 1$, by (3.2.1 a), and such that:

$$
\sigma(\psi, \varphi)=s\left(D_{s} \psi, \varphi\right), \quad \psi, \varphi \in H .
$$

Let $J\left|D_{s}\right|$ the polar decomposition of $D_{s}$; since $D_{s}$ is normal (following from the relation $D_{s}^{+}=-D_{s}$, with $D_{s}^{+}$the adjoint of $D_{s}$ with respect to $s)$, we get $\left[J, D_{s}\right]_{-}=0([20]$, p. 935). It can be easily shown [6] that a $\sigma$-allowed hilbertian structure is defined on $H$ through $J$ ([21], p. 28 and 29), i.e. if a multiplication law is defined, combining elements in $H$ with complex numbers such as:

$$
(\alpha+i \beta) \psi=\alpha \psi+\beta J \psi, \quad \alpha, \beta \in R, \quad \psi \in H .
$$

then $H$, equipped with the scalar product:

$$
h(\psi, \varphi)=s_{J}(\psi, \varphi)+i \sigma(\psi, \varphi)
$$

with $s_{J}(\psi, \varphi)=-\sigma(J \psi, \varphi), \psi$ and $\varphi \in H$, is a complex Hilbert space; it is straightforward that $s_{J} \in \widetilde{\Xi}$. 
The range $H_{s}$ of the operator $D_{s}$ is dense in $H$, while the operator $A_{s}=-D_{s}^{-1}=J\left|D_{s}\right|^{-1}$, defined on $H_{s}$, is generally unbounded. $A_{s}$ is bounded precisely when $H_{s}=H$. Since $\left\|D_{s}\right\| \leqq 1,\left|A_{s}\right| \geqq 1$, and it follows that $\left|A_{s}\right|^{-1}$ is a positive operator. Moreover, for any $s \in \mathcal{E}$, a conjugation $\Lambda$ (i.e. $[\Lambda, J]_{+}=0$ with $\Lambda^{2}=1$ ) can be found such that $\left[\left|A_{s}\right|, \Lambda\right]_{-}=0$ ([6], Prop. 8).

Remark. If $(H, h)$ is a complex Hilbert space with $\sigma$ as the imaginary part of the scalar product $h$, it is well known that $(H, \sigma)$ is sequentially closed. From above we deduce that whenever $(H, \sigma)$ is a symplectic sequentially closed space, $\sigma$-allowed hilbertian structures can be found provided that $\subseteq$ be not empty.

\subsection{On the Product Form of Quasi-Free States}

It is shown in [6] that any quasi-free state $\omega$ on $\overline{\Delta(H, \sigma)}$ is defined by:

$$
\omega\left(\delta_{\psi}\right)=\exp \left\{i \chi(\psi)-\frac{1}{2} s(\psi, \psi)\right\}, \quad \psi \in H
$$

where $\chi$ is in the algebraic dual of $H$, and $s$ is a real scalar product on $H$ verifying (3.2.1 a). We are loocking only for Von Neumann algebras generated through the representations $\pi_{\omega}$ corresponding to quasi-free states via the Gelfand-Segal theorem. Since $\omega=\omega_{s} \circ \xi_{\chi}$ with $\omega_{s}$ such that:

$$
\omega_{s}\left(\delta_{\psi}\right)=\exp \left\{-\frac{1}{2} s(\psi, \psi)\right\} .
$$

$\xi_{\chi}$ being the gauge automorphism of second kind induced by $\chi$, the study of the quasi-free states such as $\omega_{s}$ is only needed. Moreover it is shown in ([6] th. 3, Prop. 11) that $\omega_{s}$ is primary precisely when $s \in \mathcal{S}$. From now on, only these states will be examined. $\pi_{s}, \mathfrak{H}_{s}$ and $\Omega_{s}$ denote the representation, the space and the cyclic vector obtained from $\omega_{s}$ through the Gelfand-Segal theorem.

When $\left\|D_{s}\right\|=1$ [i.e. when $D_{s}$ is a $\sigma$-allowed hilbertian structure on $(H, \sigma)], \omega_{s}$ is called "Fock state", known as a pure one.

(3.3.1) Theorem. For any $s \in \mathfrak{S}, H_{1}$ being the kernel of $\left|D_{s}\right|-1, H_{2}$ the orthocomplement of $H_{1}$ in $H$ through $s\left[\right.$ so that $\overline{\Delta(H, \sigma)}=\overline{\Delta\left(H_{1}, \sigma\right)}$ $\left.\otimes \overline{\Delta\left(H_{2}, \sigma\right)}\right]$, then $\omega_{s}=\omega_{1} \otimes \omega_{2}$, with $\omega_{i}=\omega_{s} \mid \overline{\Delta\left(H_{i}, \sigma\right)}, i=1,2$. Moreover $\omega_{1}$ is pure, but $\omega_{2}$ is not pure.

The first part follows from the decomposition:

$$
\begin{gathered}
\psi=\psi_{1}+\psi_{2} \text { with } \psi_{i} \in H_{i}, \quad i=1,2, \text { for any } \psi \in H, \\
\text { with } \\
\|\psi\|_{s}^{2}=\left\|\psi_{1}\right\|_{s}^{2}+\left\|\psi_{2}\right\|_{s}^{2}
\end{gathered}
$$

and from its direct consequence

$$
\omega_{s}\left(\delta_{\psi}\right)=\omega_{1}\left(\delta_{\psi_{1}}\right) \omega_{2}\left(\delta_{\psi_{2}}\right) .
$$

$\omega_{1}$ is a Fock state (consequently pure) on $\overline{\Delta\left(H_{1}, \sigma\right)}$ since $D_{s}\left|H_{1}=J\right| H_{1}$. 
The proof is achieved through the following lemma:

(3.3.2) Lemma. For any $s \in \mathcal{G}$, if $\operatorname{ker}\left(\left|D_{s}\right|-1\right)=\{0\}, \omega_{s}$ is not pure.

The image $H_{s}$ of $H$ through $D_{s}$ is everywhere dense, and the mapping $\psi \in H \rightarrow \pi_{s}\left(\delta_{\psi}\right) \in \mathfrak{L}\left(\mathfrak{S}_{s}\right)$ strongly continuous, the proof that $\omega_{s} \mid \Delta\left(H_{s}, \sigma\right)$ is not pure will be sufficient ([6], Prop. 5). Take the operators:

$$
\begin{aligned}
& T_{1}=\frac{1}{\sqrt{2}}\left(\left|A_{s}\right|+1\right)^{1 / 2} \\
& T_{2}=\frac{1}{\sqrt{2}}\left(\left|A_{s}\right|-1\right)^{1 / 2}
\end{aligned}
$$

defined on $H_{s}$, with a range dense in $H$; this last property is straightforward as far as $T_{1}$ is concerned, and is easily deduced for $T_{2}$ from the hypothesis of the lemma. It implies, just as in ([22], p. 648), that the representation $\pi$ of $\overline{\Delta\left(H_{s}, \sigma\right)}$ in $\mathfrak{G}_{s_{J}} \otimes \mathfrak{G}_{\delta_{J}}$ defined by:

$$
\pi\left(\delta_{\psi}\right)=\pi_{s_{J}}\left(\delta_{T_{1} \psi}\right) \otimes \pi_{s_{J}}\left(\delta_{T_{2} \psi}\right), \quad \psi \in H_{s}
$$

has $\Omega_{s_{J}} \otimes \Omega_{s_{J}}$ as cyclic vector.

The representation $\pi^{\prime}$ of $\overline{\Delta\left(H_{s}, \sigma\right)}$ into $\mathfrak{G}_{s_{J}} \otimes \mathfrak{G}_{s_{J}}$ defined by:

$$
\pi^{\prime}\left(\delta_{\psi}\right)=\pi_{s_{J}}\left(\delta_{T_{2} \psi}\right) \otimes \pi_{s_{J}}\left(\delta_{T_{1} \psi}\right), \quad \psi \in H_{s}
$$

has $\Omega_{s_{J}} \otimes \Omega_{s_{J}}$ too as cyclic vector, and commutes with $\pi$. Consequently $\pi$ is a reducible representation; since:

$$
\omega_{s}\left(\delta_{\psi}\right)=\left(\Omega_{s_{J}} \otimes \Omega_{s_{J}} \mid \pi\left(\delta_{\psi}\right) \Omega_{\delta_{J}} \otimes \Omega_{s_{J}}\right)
$$

$\omega_{s} \overline{\Delta\left(H_{s}, \sigma\right)}$ is not pure.

(3.3.5) Corollary. The pure states $\omega_{s}$ are the Fock states.

Indeed, from $([17], 2.2)$ the tensor-product states are pure precisely when each component state is pure.

\subsection{Invariant Primary Quasi-Free States}

We denote $\mathcal{L}^{2}\left(R^{3}\right)$ by $H$, and by $\sigma$ the imaginary part of the usual scalar product. The translation group $\left\{T_{x} \mid x \in R^{3}\right\}$ is a group of symplectic operators inducing [see (3.1.1)] a group of automorphisms $\left\{\tau_{x} \mid x \in R^{3}\right\}$ of $\overline{\Delta(H, \sigma)}$ defined by:

$$
\tau_{x}\left(\delta_{\psi}\right)=\delta_{T_{x} \psi}, \quad \psi \in H .
$$

A quasi-free state $\omega_{s}, s \in \mathbb{E}$, is translation-invariant as soon as, for any $x \in R^{3}, \tau_{x}$ is orthogonal with respect to $s$, or equivalently:

$$
\left[\tau_{x}, D_{s}\right]_{-}=0 \text { for any } x \in R^{3} .
$$

This can be restated by saying that $\tau_{x}$ and $A_{s}$ commute on the domain of $A_{s}$. Looking at translation-invariant quasi-free states will allow us to make the results of preceding sections more precise. In particular, we get the following theorem: 
(3.4.1) Theorem. The notations being as in Theorem (3.3.1), if $\omega_{s}$ is translation invariant, we get $\omega_{s}=\omega_{1} \otimes \omega_{2}$ with:

$\omega_{1}$ pure of type $I_{\infty}$,

$\omega_{2}$ primary of type $I I I$.

Hence, if $H_{2} \neq\{0\}, \omega_{s}$ is primary of type $I I I$ ([10], p. 102, Cor. 3).

$\omega_{1}$ is known to be pure, hence primary of type $I_{\infty}$. The following lemma is only needed:

(3.4.2) Lemma. For any $s \in \subseteq$ such that $H_{1}=\{0\}$, if $\omega_{s}$ is invariant it is a type III state.

Arguing as in Lemma (3.3.2), the representation $\pi$ (3.3.3) must be shown to be a primary one, of type III. $\Omega_{s_{J}} \otimes \Omega_{s_{J}}$ is cyclic for $\pi$ and separating for $\{\pi \overline{(\Delta(H, \sigma)})\}^{\prime \prime}$, since it is cyclic for $\pi^{\prime}$ (3.3.4). From ([16], Theorem 2.4), the result follows by establishing that $\Omega_{s_{J}} \otimes \Omega_{s_{J}}$ is the unique invariant vector. From ([15], Theorem 1), we get it from:

Now

$$
\omega_{s}\left(\delta_{\psi} \delta_{T_{x} \varphi}\right)-\omega_{s}\left(\delta_{\psi}\right) \omega_{s}\left(\delta_{\varphi}\right) \rightarrow 0 \text { with }|x| \rightarrow \infty
$$

and

$$
\omega_{s}\left(\delta_{\psi} \delta_{T_{x} \varphi}\right)=\omega\left(\delta_{\psi}\right) \omega\left(\delta_{\varphi}\right) e^{-\left(s\left(\psi, T_{x} \varphi\right)+i \sigma\left(\psi, T_{x} \varphi\right)\right)}
$$

$$
\lim _{|x| \rightarrow \infty}\left(s\left(\psi, T_{x} \varphi\right)+i \sigma\left(\psi, T_{x} \varphi\right)\right)=0
$$

using ([13], 14.10.7) just as in the Lemma (2.3.7). The Theorem (3.4.1) is now derived.

Translation-invariance of quasi-free states also allows us to state precisely the structure of $D_{s}$ and $A_{s}$. In particular, using the notations of (3.3.1) [and as in the fermion case (2.3.6)], $H_{1}$ and $H_{2}$ can be shown to have zero or infinite dimension. Moreover, as in ([5], Section IV), the bounded operator $D_{s}, s \in \mathcal{G}$, is such that, for any $\varphi \in \mathcal{I}^{2}\left(R^{3}\right)$

$$
\left(\widetilde{D_{s} \varphi}\right)(p)=i d_{1}(p) \tilde{\varphi}(p)+d_{2}(p) \overline{\tilde{\varphi}(-p)}, \quad p \in R^{3} \text {. }
$$

$\tilde{\varphi}$ denoting the Fourier-transform of $\varphi \in \mathcal{I}^{2}\left(R^{3}\right)$, with $d_{1}$ a real function and $d_{2}$ a symmetrical one (see [9] for the calculations).

Properties (3.2.1) are equivalent to the following relations, which are holding almost everywhere:

with

$$
\begin{aligned}
d_{1}(p) & \geqq d(p)>0, \\
1-d(p) & \geqq d_{1}(p)-d_{1}(-p),
\end{aligned}
$$

$$
d(p)=d_{1}(p) d_{1}(-p)-\left|d_{2}(p)\right|^{2} .
$$

With the same notations, the operator $A_{s}$ is such that, for any $\varphi$ in its domain and for almost every $p$ :

$$
\widetilde{\left(A_{s} \varphi\right)}(p)=i a_{1}(p) \tilde{\varphi}(p)+a_{2}(p) \overline{\tilde{\varphi}(-p)}
$$


with

$$
a_{1}(p)=d_{1}(-p) / d(p), \quad a_{2}(p)=d_{2}(p) / d(p) .
$$

Using (3.4.3), it can be easily seen that any invariant quasi-free state $\omega_{s}, s \in \mathfrak{S}$, can be characterized with the following relations which hold almost everywhere:

$$
\begin{aligned}
a_{1}(p) & \geqq 1, \\
a_{1}(p) & =a_{2}(-p), \\
\left(a_{1}(-p)+1\right)\left(a_{1}(p)-1\right)-\left|a_{2}(p)\right|^{2} & \geqq 0 .
\end{aligned}
$$

To compare these latter relations with the corresponding ones in [2] would be interesting.

\section{Conclusion}

Many factors of type III have been exhibited through the study of invariant quasi-free states of the $C^{*}$-algebras of commutation and anticommutation relations. In particular, for fermions, the quasi-free states $\omega_{2 \lambda J}$ [where $J$ means multiplication by $i$ in the space $\left.\mathfrak{L}^{2}\left(R^{3}\right)\right], 0<\lambda<1 / 2$, are the states shown by Powers to be algebraically inequivalent [3].

Nevertheless, to classify and study invariant quasi-free states as in the sections above, is not only mathematically interesting. Therefore ARAKI and Wrss, describing the equilibrium state of the Fermi gas [23], get, for zero temperature, a Fock state and, for finite temperature, a quasi-free state $\omega_{A}$ which is primary of type III, and invariant through translations and through the gauge-group. It can be defined as follows: $\varrho$ being the density in the momentum space, $A$ is the operator

$$
\widetilde{(A f)}(p)=i(1-2 \varrho(p)) \tilde{f}(p), f \in \mathfrak{Q}^{2}\left(R^{3}\right) .
$$

Analogously, in the Bose case [22], Arakr and Woods get at finite temperature, when the fundamental state is not macroscopically occupied, a quasi-free state $\omega_{s}$ which is invariant through translations and through the gauge group; it is defined as follows:

$$
\widetilde{\left(A_{s} f\right)}(p)=i(1+2 \varrho(p)) \tilde{f}(p), \quad f \in \mathfrak{I}^{2}\left(R^{3}\right) .
$$

When the fundamental state is macroscopically occupied, and particularly at zero-temperature, we get states which are no longer quasifree, but Hilbert sums of such quasi-free states.

Acknowledgements. The authors are very indebted to Professor D. KASTLER for constant interest in this paper and fruitful help during its preparation.

They are also indebted to Professors A. Grossman, A. Gurchardet, N. Hugenholtz, A. Kirillov, M. Sirugue, E. Størmer, J. C. Trotin and M. Winnink for illuminating discussions. 


\title{
References
}

1. Greenberg, O. W.: Ann. Phys. 16, 158 (1961).

2. Robinson, D. W.: Commun. Math. Phys. 1, 159 (1965).

3. Powers, R. T.: Princeton Thesis (1967).

4. Balslev, E., and A. Verbeure: Commun. Math. Phys. 1, 55 (1968).

5. - J. Manucead, and A. Verbeure: Commun. Math. Phys. 8, 315 (1968).

6. Manuceau, J., and A. Verbeure: Commun. Math. Phys. 9, 293 (1968).

7. Shale, D., and W. F. Strnespring: Ann. Math. 80, 365 (1964).

8. Gurchardet, A.: Algèbres d'observables associées aux relations de commutation (to appear).

9. Séminaire du Centre de Physique Théorique de Marseille (1968).

10. Dixmrer, J.: Les algèbres d'opérateurs dans l'espace hilbertien. Paris: GauthierVillars 1958.

11. - Les $C^{*}$-algèbres et leurs représentations. Paris: Gauthier-Villars 1964.

12. Powers, R. T.: Ann. Math. (1967).

13. Dieudonne, J.: Eléments d'analyse, tome 2. Paris: Gauthier-Villars 1968.

14. Godement, R.: Trans. Am. Math. Soc. 63, 1 (1948).

15. Kastrler, D., and D. W. Robinson: Commun. Math. Phys. 3, 151 (1966).

16. Størmer, E.: Commun. Math. Phys. 6, 194 (1967).

17. Guichardet, A.: Ann. Sci. E.N.S. 83, 1 (1966).

18. Størmer, E.: Symmetric states of infinite tensor products of $C^{*}$-algebras (preprint).

19. Manucead, J.: Ann. Inst. Henri Poincaré 2, 139 (1968).

20. Dunford, N., and J. T. Schwartz: Linear operators II. New-York: Interscience Publ. Inc. 1963.

21. Kastrer, D.: Commun. Math. Phys. 1, 14 (1965).

22. Arakr, H., and E. J. Woods: J. Math. Phys. 4, 637 (1963).

23. - , and W. WYss: Helv. Phys. Acta 37, 136 (1964).

\author{
J. Manuceau \\ F. ROCCA \\ D. Testard \\ Centre de Physique Théorique \\ 31, Chemin Joseph Aiguier \\ F 13-Marseille $\left(9^{e}\right)$
}

